\title{
Heart Rate Turbulence in Children-Age and Heart Rate Relationships
}

\author{
MAREK KOWALEWSKI, MAREK ALIFIER, DARIUSZ BOCHEN, AND MIROSŁAWA URBAN
}

\begin{abstract}
Second Department of Pediatrics [M.K., M.U.], Medical University in Biatystok, Children's Hospital, 15-274 Biatystok, Poland; Department of Neonatology [M.A.], Medical University in Biatystok, Public Clinic Hospital, 15-276 Biatystok, Poland; Faculty of Electrical Engineering [D.B.], Technical University of Białystok, 15-351 Biatystok, Poland
\end{abstract}

\begin{abstract}
Heart rate turbulence (HRT) is a chronotropic response of the sinus heart rhythm to ventricular premature beat (VPB). Children show decreasing heart rate together with the maturation of the autonomic nervous system. The aim of the research was to assess the relationship between HRT parameters, age, and heart rate and time domain heart rate variability (HRV) parameters in healthy children. Twenty-four-hour ECG Holter recording was performed on 398 healthy children. The mean RR interval preceding VPB, number of VPBs, and HRT parameters-turbulence onset (TO) and turbulence slope (TS)—were determined. We observed significant correlation among TS and mean RR and age. Children with prepubertal status have lower values of TS compared with those during puberty. According to given quartiles, upper for TO was $\geq-0.8 \%$, lower for TS was $\leq 4.56 \mathrm{~ms} / \mathrm{RR}, 13$ patients $(3 \%)$ obtained abnormal both TO and TS. The correlations between HRT and HRV parameters were observed among the youngest children. Age and heart rate preceding VPB have no effect on HRT onset in children, whereas HRT slope is highly dependent on these variables. Our results support hypothesis that in older children HRT is dependent on autonomic tone and also determined by other intrinsic modulators. (Pediatr Res 62: 710-714, 2007)
\end{abstract}

$\mathrm{T}$ he term "heart rate turbulence" (HRT) was first used by Schmidt et al. (1) and refers to changes in the sinus heart rhythm after ventricular premature beat (VPB). The response consists of a short phase of early heart rate acceleration followed by heart rate deceleration. HRT can be quantified by two parameters, turbulence onset (TO) and turbulence slope (TS), which are strong and independent markers of a sudden cardiac death risk in various patients' cohorts $(1,2)$. The underlying mechanism of described parameters is connected with compensatory activation of baroreceptor reflex triggered by a decreased stroke volume following a VPB. The HRT response is believed to be under strong dependence on parasympathetic activity and heart rate preceding VPB (3). Recent studies have shown that HRT phenomenon can be used as an alternative measure of baroreflex sensitivity $(4,5)$.

A few studies demonstrate that in healthy subjects increasing age is associated with a decrease of HRT slope $(6,7)$, likewise an increase in heart rate is relevant to TO decrease. However, there is a lack of information about heart rate

Received March 21, 2007; accepted July 2, 2007.

Correspondence: Marek A. Kowalewski, M.D., Ph.D., Second Department of Pediatrics, Medical University in Białystok, Children's Hospital, Waszyngtona 17 str, 15-274 Białystok, Poland; e-mail: m.a.kowalewski@wp.pl turbulence behavior after VPB and the potential influence of age and pubertal status on healthy subjects within first two decades of life. Changes in basic heart rhythm during child's development are influenced by breathing frequency, sleeping periods and maturation of sympathetic and parasympathetic systems (8). We hypothesized that in such population age heart rhythm may significantly influence the heart rate turbulence parameters.

The aim of this study was to determine the correlation among the HRT parameters, age, heart rate, heart rate variability (HRV) parameters and pubertal status in healthy children.

\section{MATERIALS}

All the participants had a documented history of ventricular arrhythmia and were included in the study at the Cardiology Outpatient Clinic. The research involved 410 children (200 boys and 210 girls) aged between 1 mo and $19 \mathrm{y}$. Informed consent has been obtained from the parents or the legal guardians of all included children or, if the patient was older than 18, from the patient themselves. The study protocol has been approved by the Medical University of Bialystok Ethics Committee.

The group was matched so that each yearlong interval had a similar number of children (on average 20) with nearly equal percentage of boys and girls.

The inclusion criteria were sinus rhythm with absence of any intraventricular conduction disorders in 12-lead ECG before the study, normal values of resting blood pressure, and normal results of transthoracic echocardiography without evidence of congenital heart defect. Patients who had a history of chronic diseases and cardiac surgery, or at least 3 mo before and at the time of the study had received any medications that might potentially interfere with autonomic system were excluded from the study. The pubertal status was assessed according to the Tanner scale. To assess the influence of age and pubertal status on HRT parameters, we divided patients into groups: (A) up to $1 \mathrm{y}$, (B) from 13 mo to the beginning of puberty (up to $\mathrm{I}^{\mathrm{O}}$ Tanner scale), and (C) subjects who were either in at least $\mathrm{II}^{\mathrm{O}}$ of puberty (according to Tanner) or in the postpubertal age up to 19th year of life.

All children had Holter recordings using a digital three-channel recorder with a sampling rate of $1 \mathrm{kHz}$ (FD-3, Oxford Instruments Ltd., Abington, UK). The obtained results were blinded and assessed manually with the Oxford Excel 2 program (Oxford Instruments, Ltd.). The Holter recording was independently assessed by two investigators for exclusion of artifacts. A minimum of $23 \mathrm{~h}$ of recording and at least five ventricular premature beats were required for a 24-h ambulatory recording to be acceptable (1).

Patients with only monomorphic premature beats were assessed according to the protocol. When polymorphic or complex form of arrhythmia (classified higher as Lown III) occurred in Holter recording, the child was excluded from the study group. No children with couplets or runs were included in the study. Whenever in Holter recording we found the atrial premature beat with

Abbreviations: HRT, heart rate turbulence; TO, turbulence onset; TS, turbulence slope; VPB, ventricular premature beat 
aberration similar to the ventricular premature beat, the whole recording was excluded from further evaluation.

$\boldsymbol{H R} \boldsymbol{V}$ analysis. Time-domain HRV parameters were measured according to the Task Force of the European Society of Cardiology and North American Society of Pacing and Electrophysiology (9). The following 24-h measurements were made: SD of NN-intervals (SDNN), mean SD of NN-intervals for all 5-min segments (SDNNi), SD of the averages of NN-intervals for all 5-min segments (SDANN), root mean square of difference of consecutive normalto-normal intervals (rMSSD), and geometric triangular index (HRVTI; i.e. the integral of the density distribution divided by the maximum density distribution).

HRT analysis. HRT was not analyzed if VPB was preceded by the artifacts or by premature beats within $20 \mathrm{RR}$ subsequent intervals. The method used in the analysis consisted of two parameters: TO and TS. TO was defined as the relative difference between the mean of the first two sinus RR intervals following VPB and the mean of two RR intervals preceding VPB. Turbulence slope was defined as the maximum positive slope of a regression line assessed over any sequence of five subsequent sinus rhythm RR intervals within the first 20 sinus rhythm intervals after VPB. TO was calculated separately for each VPB and then averaged, whereas TS was calculated based on local algorithm averaging. The RR intervals with following characteristic $<200 \mathrm{~ms}$ and $>2000 \mathrm{~ms}$, with jumps $>200 \mathrm{~ms}$ from one interval to the next, were excluded from the heart rate turbulence analysis (10). Additionally, VPBs with too long coupling intervals or too short compensatory pause were rejected (the HRT calculation was limited to VPBs with a minimum prematurity of $20 \%$ and a postextrasystole interval which was at least $20 \%$ longer than the reference interval (mean of the five last sinus intervals preceding VPB) (11).

Statistical analysis. All continuous variables were expressed as mean \pm SD for variables of normal distribution and as median for skewed distribution. Correlations between parameters with normal distribution were calculated using Pearson's correlation coefficient. When skewed distributed data were observed, Spearman's rank correlation was applied. To assess abnormal TO values, the upper quartile was used, whereas in the case of TS the lower quartile was applied. The comparison among mean values in the group was carried out with Mann-Whitney's $U$ test with $p$ value $<0.05$ considered as statistically significant. All statistical calculations were performed using SPSS 9 (SPSS Inc., Chicago, IL).

\section{RESULTS}

From the initially 410 enrolled Holter recordings, only 398 met the criteria of the study. The number of VPBs varied between 5 and 9568 (median 102 VPBs) per one recording. Mean RR interval was between $0.432 \mathrm{~s}$ and $1.230 \mathrm{~s}$ (Table 1). The values of the HRV parameters were significantly different among groups (Table 1). For the whole group, HRT parameters varied between $-28.4 \%$ and $7.0 \%$ for TO and between 0.47 and $23.5 \mathrm{~ms} / \mathrm{RR}$ for TS. The upper quartile for TO was $\geq-0.8 \%$ and lower quartile for TS was $\leq 4.56 \mathrm{~ms} / \mathrm{RR}$. According to given quartiles, 65 (16\%) of the studied children had abnormal TO or TS (one from group A, 38 from group B and 26 from group C), and 13 (3\%) obtained both TO and TS abnormal (0 from group A, 9 from group B, and 4 from group C).

In respective groups, upper quartile for $\mathrm{TO}$ and lower quartile for TS were as follows: group A: TO $\geq-2.0 \%$, TS $\leq 2.20 \mathrm{~ms} / \mathrm{RR}$, group B: TO $\geq-1.0 \%$, TS $\leq 3.70 \mathrm{~ms} / \mathrm{RR}$, and group C: TO $\geq-0.9 \%$, TS $\leq 6.90 \mathrm{~ms} / \mathrm{RR}$. Therefore, according to given quartiles in group A, four (19\%) of that children had abnormal TO or TS, and one (2\%) obtained both TO and TS abnormal. In group B, 48 (24\%) of them had abnormal TO or TS, and $10(5 \%)$ obtained both TO and TS abnormal. In group C, abnormal TO or TS was found in 30 (16\%) children, and $5(2.8 \%)$ gained both TO and TS abnormal.

Considering the whole study group, we could not confirm statistically significant correlation between TO and HRV parameters (SDNN $r=-0.05$, SDNN index $r=-0.07$, SDANN $r=-0.03$, RMSSD $r=-0.05$, HRTI $r=-0.11$ ). However, we found such correlations between TS and SDNN and SDANN (respectively, $r=0.22, p<0.01$ and $r=0.21, p<$ $0.01)$. For SDNN index, RMSSD and HRTI correlations with TS were not significant (respectively, $r=0.12, r=0.11, r=$ $0.13)$.

In study group A the majority of HRV parameters show positive correlations with TO and TS (Table 2). In study group $\mathrm{B}$, only three of them (SDNN index, RMSSD, and HRTI) revealed significant correlation with TO. No such dependencies were found in study group C (Table 2).

The correlations between age, mean RR interval, and the indices of HRT are shown in Table 2 and Figures 1-4.

Table 1. Baseline characteristic of analyzed data

\begin{tabular}{|c|c|c|c|c|}
\hline & All subjects $(n=398)^{*}$ & Group A $(n=21)$ & Group B $(n=199)$ & Group C $(n=178)$ \\
\hline \multicolumn{5}{|c|}{ Demographic and Holter recordings data } \\
\hline Age $(\mathrm{y})$ & $8.7 \pm 5.7$ & $0.65 \pm 0.3$ & $6.2 \pm 2.8$ & $15.2 \pm 2.0$ \\
\hline Boys/girls & $193 / 205$ & $10 / 11$ & $100 / 99$ & $83 / 95$ \\
\hline $\begin{array}{l}\text { Duration of Holter } \\
\text { recordings (h) }\end{array}$ & $23.5 \pm 0.2$ & $23.3 \pm 0.1$ & $23.6 \pm 0.2$ & $23.4 \pm 0.1$ \\
\hline VPB (total count) & $587 \pm 1588(102)$ & $859 \pm 1628(99) \dagger$ & $372 \pm 947(62) \ddagger$ & $204 \pm 956(57)$ \\
\hline $\mathrm{VPB} / \mathrm{h}$ & $26 \pm 72(3.3)$ & $37 \pm 89(4.1)$ & $18 \pm 52(2.9)$ & $10 \pm 54(1.8)$ \\
\hline Mean RR interval (s) & $0.666 \pm 0.125$ & $0.494 \pm 0.046 \S$ & $0.650 \pm 0.082 \dagger$ & $0.765 \pm 0.11$ \\
\hline \multicolumn{5}{|l|}{ HRV parameters } \\
\hline SDNN (ms) & $150 \pm 57$ & $106 \pm 42 \S$ & $139 \pm 48 \dagger$ & $181 \pm 60$ \\
\hline SDNN index (ms) & $90 \pm 41$ & $65 \pm 29 \S$ & $85 \pm 29 \dagger$ & $108 \pm 52$ \\
\hline SDANN (ms) & $110 \pm 47$ & $76 \pm 37 \S$ & $102 \pm 45 \dagger$ & $135 \pm 42$ \\
\hline RMSSD (ms) & $110 \pm 75$ & $87 \pm 51 \dagger$ & $106 \pm 56$ & $124 \pm 102$ \\
\hline HRVTI & $16 \pm 7$ & $11 \pm 4 \S$ & $14 \pm 6 \dagger$ & $21 \pm 8$ \\
\hline \multicolumn{5}{|l|}{ HRT parameters } \\
\hline TO $(\%)$ & $-3.17 \pm 3.64(-2.93)$ & $-3.75 \pm 2.63(-3.30)$ & $-3.01 \pm 4.81(-2.90)$ & $-2.81 \pm 3.71(-3.33)$ \\
\hline $\mathrm{TS}(\mathrm{ms} / \mathrm{RR})$ & $9.45 \pm 6.64(8.86)$ & $7.43 \pm 6.26(4.50) \dagger, \mathbb{A}$ & $8.59 \pm 5.32(7.30) \dagger$ & $11.3 \pm 7.86(10.05)$ \\
\hline
\end{tabular}

$*$ Data shown as mean \pm SD. If the data had a skewed distribution, the median value was given in brackets. Total count $=$ the number of VPBs per Holter recording; $\mathrm{VPB} / \mathrm{h}=$ total number of $\mathrm{VPBs}$ divided by duration of recording in hours.

$\dagger p<0.001$ compared with group $\mathrm{C} ; \ddagger p<0.01$ compared with group $\mathrm{A}$ and $\mathrm{C}$; $\S p<0.001$ compared with group $\mathrm{B}$ and $\mathrm{C}$; $\mathbb{I} p<0.05$ compared with group B. 
Table 2. The results of correlation between HRT and HRV parameters and age and reference RR interval in the studied groups

\begin{tabular}{|c|c|c|c|c|c|c|}
\hline & \multicolumn{2}{|c|}{ Group A $(n=21)$} & \multicolumn{2}{|c|}{ Group B $(n=199)$} & \multicolumn{2}{|c|}{ Group C $(n=178)$} \\
\hline Age & $-0.11(0.10)$ & $0.17(0.05)$ & $-0.11(0.16)$ & $0.26(0.001)$ & $0.05(0.54)$ & $0.22(0.003)$ \\
\hline SDNN & $0.31(0.05)$ & $0.42(0.005)$ & $-0.13(0.09)$ & $0.09(0.19)$ & $-0.09(0.28)$ & $0.12(0.28)$ \\
\hline SDNN index & $0.61(0.001)$ & $0.19(0.21)$ & $-0.19(0.05)$ & $0.07(0.28)$ & $-0.07(0.43)$ & $0.06(0.45)$ \\
\hline SDANN & $-0.01(0.91)$ & $0.51(0.001)$ & $-0.05(0.43)$ & $0.09(0.19)$ & $-0.09(0.28)$ & $0.14(0.10)$ \\
\hline
\end{tabular}

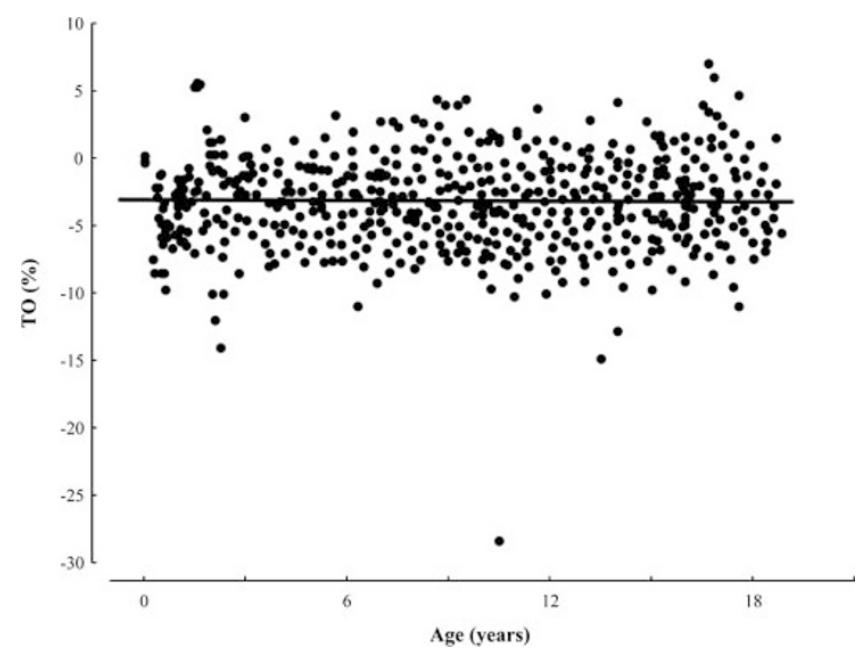

Figure 1. Correlation between TO and age in the studied group $(p=0.13$, $r=-0.010)$

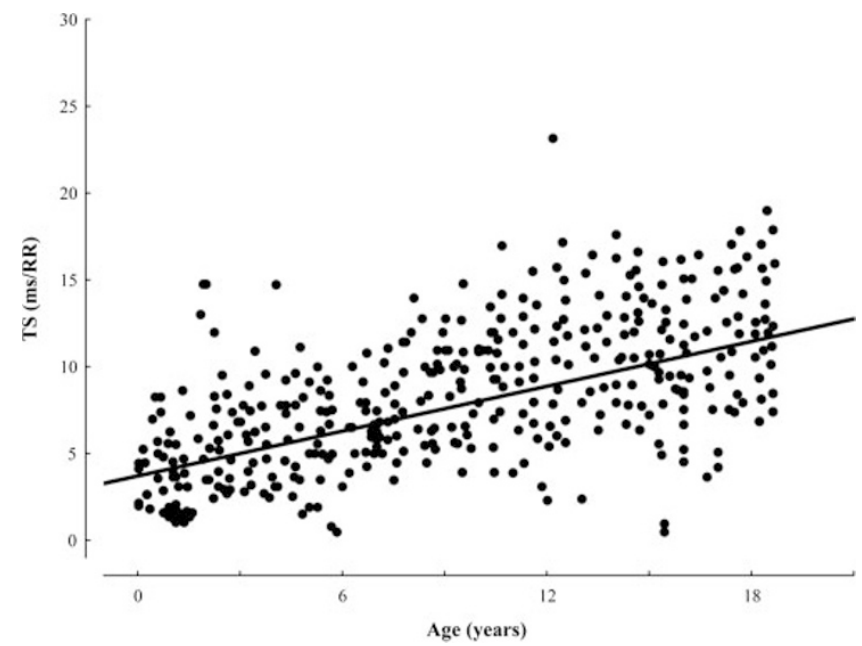

Figure 2. Correlation between TS and age in the studied group $(p<0.001$, $r=0.53$ ).

\section{DISCUSSION}

Our research shows that the turbulence slope strongly depends on age and heart rate preceding VPB in children, whereas turbulence onset is independent of these variables. Moreover, the amount of VPBs (if there are more than four VPBs during 24-h Holter recording) does not correlate with TS or TO.

It is known that the pediatric population shows a progressive maturation of the autonomic nervous system during child-

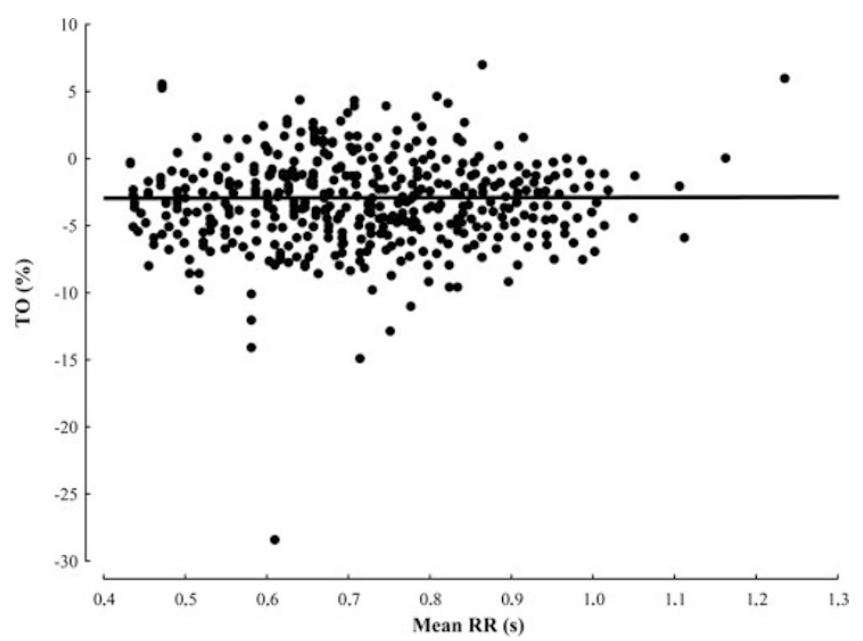

Figure 3. Correlation between $\mathrm{TO}$ and reference RR interval in the studied group $(p=0.37, r=-0.05)$.

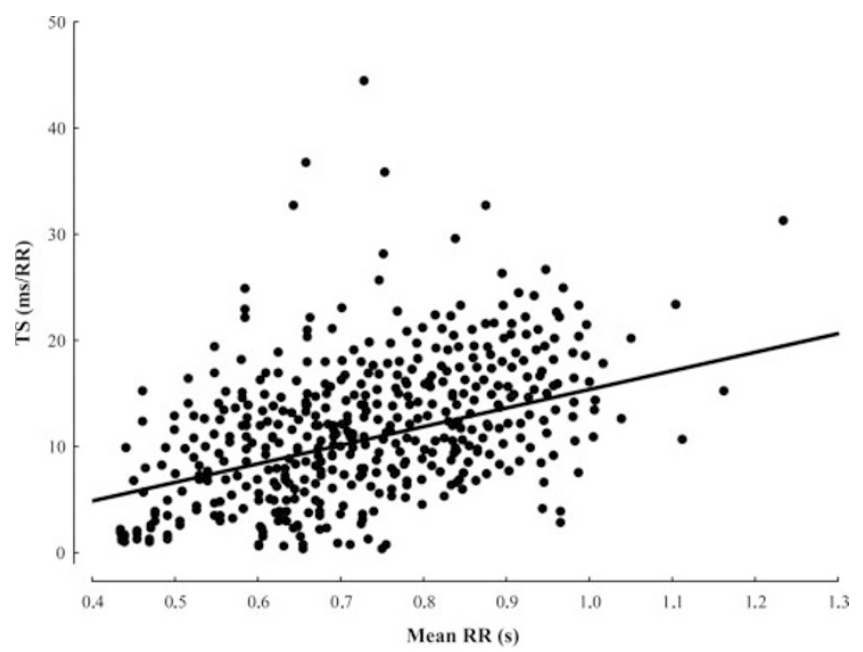

Figure 4. Correlation between TS and reference RR interval in the studied group ( $p=<0.001, r=0.32$ ).

hood. That phenomenon is associated with a shortened and different organization of sleep period, sympathetic withdrawal and parasympathetic system predominance, thus, resulting in gradually decreasing heart rate. Thereby, measures of cardiac autonomic activity (e.g. HRV parameters) have strong correlation with the mean RR interval over the length of the analysis (8). HRT is believed to be under control of the autonomous nervous system. The indices of HRT are closely linked to arterial baroreceptor reflex which were shown by 
using physiologic model involving the pressure stimulation of aorta, a baroreflex feedback by studying the dynamicity of heart rate and blood pressure response to ectopic beats in patients with congestive heart disease $(5,12)$. Thus, the heart rate turbulence indices should demonstrate the substantial correlation with age and heart rate. Our findings support the hypothesis that TS is very closely connected with both heart rate and age in analyzed groups. This remark proves that when the mean heart rate decelerates during growing, the response for VPB strengthens.

However, we must emphasize that, as opposed to others studies, we correlate the parameters of HRT to mean HR. Mean RR was chosen to avoid potential errors associated with counting of HR before VPB. Recently, Schwab et al. (7) found correlation between TS and RR intervals preceding VPB in healthy adult persons, although their statistical strength were weaker than in our study. They showed that TS slope decreased with increasing basic heart rate preceding VPB. Also, the research conducted by Grimm et al. (6) was concordant with the above findings. We presumed that decreasing heart rate during child's growing causing that turbulence slope was positively correlated with it. Our results have confirmed that hypothesis. The opposite results have been published by the others (6,7). Schwab et al. (7) and Grimm et al. (6) found in adults decreasing TS slope with increasing age. We think that both results should be taken together and they show the gradual progress of maturation and ageing of autonomic nervous system.

Contrary to TS, in our study turbulence onset did not correlate with heart rate and age. Our data are not compliant with those of Schwab et al. (7), who found significant correlations among age, heart rate, and TO in healthy persons. In their study, TO increased with increasing age and increasing heart rate, otherwise the values of TO become more positive. A possible explanation might be older age of the examined subjects in the mentioned study. The other research did not confirm similar correlations (6); however, it also included middle-aged subjects. The second factor, which could influence the results, might be a different heart rate preceding VBPs. The next possible difference is values of blood pressure, which gradually increase during development. As proved in the study conducted by Raj et al. (13), HRT parameters are physiologically modulated by the duration of the initial hypotension. We think that further research is needed to explain that discrepancy.

The parameters of HRV evolve with age and reflect the influence of the autonomic nervous system on the heart, resulting in a fluctuation of the heart rate around its mean value. It seems that in spite of several associations, parameters of HRT do not strongly correlate with those of $\operatorname{HRV}(5,12)$. Some clinical studies show only single correlations between HRT and HRV parameters; however, there are several others in the literature that indicate high correlations. Those studies were performed on adult populations in which the myocardial infarction or the coronary incidents were noted $(11,14,15)$. In our study, we found the correlations between HRT and HRV parameters predominantly occur in the youngest group of children, in which modulation of autonomic nervous system- and, hence, its influence on heart function-seems to be rather small. In older children groups, when influence of autonomic system on heart function is more expressed, we observed that correlations between HRT and HRV parameters weaken. This fact could be also supported by hypothesis that in older children and youngsters HRT is dependent not only on autonomic tone and baroreflex mechanism but on other intrinsic modulators, like mechanistic properties of the myocardium as well.

We mentioned that a mean TO achieves between $-1.6 \%$ and $-4.95 \%$, and TS between $7.1 \mathrm{~ms} / \mathrm{RR}$ to $16 \mathrm{~ms} / \mathrm{RR}$ based on studies conducted on healthy persons above $20 \mathrm{y}$ of age $(6,7,16,17)$. These values are similar to the ones presented in our study. Since TO reflects fast changes in successive sinus RR intervals, it seems to be less dependent on the heart rate $(11,18)$. In our research, there were no differences in the values of TO between the groups, which varied in the heart rate preceding VPB. Contrary to TO, TS depends on heart rate to a higher degree, hence, to assess this parameter, $20 \mathrm{RR}$ intervals must be analyzed. The hypothesis explains the fact of the higher values of TS in group C (the oldest one) with mean reference interval about $0.76 \mathrm{~s}$ in comparison to group A (the youngest one) with mean reference interval about $0.49 \mathrm{~s}$. In other words, TS tends to zero as heart rate increases.

Using defined quartile values for TS and TO, abnormal HRT results were found in 13 out of 398 (3\%) examined Holter recordings. We could not apply the previously established criteria by Schmidt et al. (1) based on EMIAT and MPIP cohort studies. The reasons are caused by the different sample age and reported diseases. Moreover, Grimm et al. (6) and Schwab et al. (7) described an incidence of false positive results in healthy middle-aged subjects when Schmidt's criteria were used as a cut-off values $(6,19)$. The incidence of $3 \%$ of abnormal results in our research is concordant with other population-based distribution indices, i.e. the prevalence of hypertension in children (20). A limitation of the study is the fact that the analyzed sample included only persons in the first two decades of life and we did not record simultaneously blood pressure with heart rhythm.

The present findings show that the first of the HRT indices, the TS, is highly dependent on age and heart rate preceding $\mathrm{VPB}$, whereas the second, the TO, is irrespective of both mentioned variables. The increase in reference interval in older children gives nearly 2 -fold higher values of TS in comparison with those who had reference interval $<0.5 \mathrm{~s}$. Higher, not lower, heart rate affected turbulence onset values. We found significant correlations of HRV and HRT parameters only in the youngest group of studied children.

This supports the hypothesis that when the autonomic nervous system matures and increases its impact on heart rate variability, its effect on HRT gradually diminishes. That might be partially explained by the fact that, in youngsters and older children, HRT is not only influenced by autonomic tone but also by other intrinsic modulators such as mechanistic properties of the myocardium.

Our study indicates that HRT parameters should be assessed in respect of heart rate and age. Further research is 
needed to investigate the utility of heart rate turbulence in prognosis of adverse outcome in pediatric population.

\section{REFERENCES}

1. Schmidt G, Malik M, Barthel P, Schneider R, Ulm K, Rolnitzky L, Camm AJ, Bigger JT Jr, Schomig A 1999 Heart-rate turbulence after ventricular premature beats as a predictor of mortality after acute myocardial infarction. Lancet 353:13901396

2. Zareba W, Moss AJ 2003 Noninvasive risk stratification in postinfarction patients with severe left ventricular dysfunction and methodology of the MADIT II noninvasive electrocardiology substudy. J Electrocardiol 36:101-108

3. Voss A, Baier V, Schumann A, Hasart A, Reinsperger F, Schirdewan A, Osterziel KJ, Leder U 2002 Postextrasystolic regulation patterns of blood pressure and heart rate in patients with idiopathic dilated cardiomyopathy. J Physiol 538:271-278

4. Lin LY, Lai LP, Lin JL, Du CC, Shau WY, Chan HL, Tseng YZ, Huang SK 2002 Tight mechanism correlation between heart rate turbulence and baroreflex sensitivity: sequential autonomic blockade analysis. J Cardiovasc Electrophysiol 13:427431

5. Davies LC, Francis DP, Ponikowski P, Piepoli MF, Coats AJ 2001 Relation of heart rate and blood pressure turbulence following premature ventricular complexes to baroreflex sensitivity in chronic congestive heart failure. Am J Cardiol 87:737-742

6. Grimm W, Sharkova J, Christ M, Schneider R, Schmidt G, Maisch B 2003 Heart rate turbulence following ventricular premature beats in healthy controls. Ann Noninvasive Electrocardiol 8:127-131

7. Schwab JO, Eichner G, Shlevkov N, Schrickel J, Yang A, Balta O, Lewalter T, Luderitz B 2005 Impact of age and basic heart rate on heart rate turbulence in healthy persons. Pacing Clin Electrophysiol 28:S198-S201

8. Massin MM, Maeyns K, Withofs N, Ravet F, Gerard P 2000 Circadian rhythm of heart rate and heart rate variability. Arch Dis Child 83:179-182

9. 1996 Heart rate variability: standards of measurement, physiological interpretation and clinical use. Task Force of the European Society of Cardiology and the North American Society of Pacing and Electrophysiology. Circulation 93:1043-1065
10. Watanabe MA, Schmidt G 2004 Heart rate turbulence: a 5-year review. Heart Rhythm 1:732-738

11. Cygankiewicz I, Wranicz JK, Bolinska H, Zaslonka J, Zareba W 2004 Relationship between heart rate turbulence and heart rate, heart rate variability, and number of ventricular premature beats in coronary patients. J Cardiovasc Electrophysiol 15:731-737

12. Mrowka R, Persson PB, Theres H, Patzak A 2000 Blunted arterial baroreflex causes "pathological" heart rate turbulence. Am J Physiol Regul Integr Comp Physiol 279:R1171-R1175

13. Raj SR, Sheldon RS, Koshman M, Roach DE 2005 Role of hypotension in heart rate turbulence physiology. Heart Rhythm 2:820-827

14. Ortak J, Weitz G, Wiegand UK, Bode F, Eberhardt F, Katus HA, Richardt G, Schunkert H, Bonnemeier H 2005 Changes in heart rate, heart rate variability, and heart rate turbulence during evolving reperfused myocardial infarction. Pacing Clin Electrophysiol 28:S227-S232

15. Ghuran A, Reid F, La Rovere MT, Schmidt G, Bigger JT Jr, Camm AJ, Schwartz PJ, Malik M 2002 Heart rate turbulence-based predictors of fatal and nonfatal cardiac arrest (The Autonomic Tone and Reflexes After Myocardial Infarction substudy). Am J Cardiol 89:184-190

16. Lindgren KS, Makikallio TH, Seppanen T, Raatikainen MJ, Castellanos A, Myerburg RJ, Huikuri HV 2003 Heart rate turbulence after ventricular and atrial premature beats in subjects without structural heart disease. J Cardiovasc Electrophysiol 14:447-452

17. Schwab JO, Shlevkov N, Grunwald K, Schrickel JW, Yang A, Lickfett L, Lewalter T, Luderitz B 2004 Influence of the point of origin on heart rate turbulence after stimulated ventricular and atrial premature beats. Basic Res Cardiol 99:56-60

18. Hallstrom AP, Stein PK, Schneider R, Hodges M, Schmidt G, Ulm K 2004 Structural relationships between measures based on heart beat intervals: potential for improved risk assessment. IEEE Trans Biomed Eng 51:1414-1420

19. Schwab JO, Eichner G, Veit G, Schmitt H, Lewalter T, Luderitz B 2004 Influence of basic heart rate and sex on heart rate turbulence in healthy subjects. Pacing Clin Electrophysiol 27:1625-1631

20. Adrogue HE, Sinaiko AR 2001 Prevalence of hypertension in junior high schoolaged children: effect of new recommendations in the 1996 Updated Task Force Report. Am J Hypertens 14:412-414 